\title{
SALUD
}

\section{Abordaje de la leishmaniasis canina en el Departamento de Alto Paraná. Zona Triple Frontera de Paraguay}

\author{
Mónica Ruoti ${ }^{1}$ Nilsa González², Andrea Giménez³ ${ }^{3}$ Cristina Arrom, \\ Jorge Miret ${ }^{4}$, Antonieta Rojas de Arias ${ }^{1}$
}

\section{Resumen}

Introducción: La leishmaniasis visceral canina (LV) es una enfermedad parasitaria sistémica grave, trasmitida por un mosquito llamado flebótomo. La epidemiología de las leishmaniasis ha sufrido modificaciones importantes en la zona de la triple frontera, considerada de emergencia y dispersión de la enfermedad. Es de notificación obligatoria para los casos de humanos y no para los caninos En la actualidad se observa un incremento de perros en los hogares, que si bien pueden influir de manera positiva en la vida de las personas pueden generar repercusiones negativas en la salud individual, pública y en el ámbito de la zoonosis. El control de los vectores está a cargo del Ministerio de Salud Pública y Bienestar Social (Programa Nacional de Control de las Leishmaniosis - SENEPA y para casos caninos el Programa Nacional de Control de Zoonosis y Centro Antirrábico Nacional (PNCZyCAN).

Objetivo: Describir aspectos relacionados con la emergencia y dispersión de las Leishmaniasis en la frontera Argentina, Brasil y Paraguay abordado en un proyecto de investigación

Material y Método: Estudio observacional descriptivo de enfoque cualitativo y cuantitativo. Primeramente se procedió al reconocimiento y observación de la zona, luego se realizaron 47 entrevistas abiertas y semi-estructuradas, a los responsables de las políticas locales, agentes encargados del manejo de sistemas de salud, área municipal de zoonosis y medio ambiente, del control de vectores en la región, protectoras de animales, criadores, acumuladores de perros (ONGs y hogares). Las entrevistas fueron en su mayoría grabadas y posteriormente transcriptas textualmente. En un tercer momento se realizó una

\footnotetext{
1. Centro para el Desarrollo de Investigación Científica-CEDIC. Universidad Nacional de Asunción, Paraguay.

2. Instituto de Investigaciones en Ciencias de la Salud. Universidad Nacional de Asunción, Paraguay.

3. Centro de Investigaciones Médicas, Universidad Nacional del Este, Paraguay.

4. Centro Antirrábico Nacional, Ministerio de Salud Pública y Bienestar Social, Paraguay.

5. Facultad de Ciencias de la Salud, Universidad Nacional del Este, Paraguay.

Proyecto "Abordando la emergencia y dispersión de la Leishmaniosis en la frontera Argentina, Brasil y Paraguay" financiado por la IDRC Canadá. Proyecto No107577-001.

E-mail: monicaruoti@gmail.com

DOI: 10.26885/rcei.foro.2017.164
} 
encuesta sobre conocimientos, actitudes y prácticas de la población en relación a las Leishmaniasis. Muestra integrada por 126 familias, pertenecientes a tres zonas de la Ciudad de Pte. Franco. Cuestionario estructurado aplicado por medio de entrevistas. Procesamiento con SPSS. Firma del consentimiento informado.

Resultados: El 73\%(92) de la población encuestada escuchó sobre la leishmaniasis, el $26.1 \%$ señaló la canina o visceral. El $73.6 \%$ no sabe cómo se transmite la enfermedad; el $58.2 \%$ no sabe qué hacer para saber si el perro está con Leishmnasiasis, solo el 6.6\% refirió que "análisis de sangre". El 64.5\% manifiesta que le sacrificaría a su perro en caso de ser diagnosticado. Solo el $8.8 \%$ lo considera como un problema en la zona. En cuanto a los actores sociales entrevistados. No se percibe en la actualidad presencia de casos confirmados, si con sospecha. En cuanto al diagnóstico y tratamiento existe conocimiento de que la prueba de Montenegro se realiza en SENEPA y que pueden solicitar el medicamento que es gratuito. En el Centro de Salud se cuenta con la ficha de notificación, no así en las Unidades de Salud de la Familia (USF) si bien se ocupan de identificar en su comunidad, casos de enfermedades, en su esquema no contempla la de leishmaniosis. En zona rural no se cuenta con laboratorios, servicios asistenciales privados, y no todas las veterinarias están en condiciones de brindar atención a los perros con leishmaniosis. El municipio del área rural tiene formado un equipo de respuesta y trabaja en coordinación con el servicio de salud y el SENEPA (MSPyBS). La principal dificultad para el diagnóstico y tratamiento es la discontinuidad del tratamiento y abandono, debido a las condiciones económicas desfavorables que dificultan el traslado de la población a la ciudad para acceder exámenes de laboratorio Identifican al SENEPA, que visitan comunidades y asentamientos de la zona rural pero trabajan más en prevención y control de otras enfermedades; realizan diagnóstico gratuito, aunque no siempre cuenta con insumos. Desde los servicios asistenciales no se realizan tareas preventivas en las comunidades. En cuanto al origen, producción y consumo, en su mayoría se obtiene de la cría en hogares, de veterinarias que lo comercializan y de organizaciones protectoras ofrecen gratuitamente en adopción. Además son traídos de Asunción, otras ciudades y de Brasil y Argentina, ya que no existe un adecuado control, lo que facilita el intercambio de animales en las fronteras.

Conclusiones: En áreas urbanas la población tiene mayor conocimiento sobre la enfermedad, aunque no muy certero. Casi todos los veterinarios de áreas urbanas la conocen, no así las de áreas rurales. Personal de salud recibió capacitación, pero no suficiente. Se percibe una actitud positiva hacia la eutanasia. Se hace necesario capacitar al personal de salud y realizar tareas educativas preventivas para la población en las comunidades, al igual que a los profesionales veterinarios. Se hace necesario fortalecer del acceso al diagnóstico temprano, tratamiento adecuado de los casos y reducción del contacto entre el hombre y el vector atendiendo a la amenaza del rebrote en los países fronterizos. Se demanda una coordinación interinstitucional para a para mejorar las condiciones de abordaje en la zona de frontera. 
Rev. cient. estud. investing. VI Foro de Investigadores; diciembre 2017

Palabras clave: leishmaniosis visceral, conocimiento, percepción, riesgo y vulnerabilidad.

\section{Referencias}

Arbo, A. (2014). Leishmaniasis visceral en Paraguay. Editorial. Rev. Inst. Med. Trop, 9(1), 4-6.

Bartlett, K. (sf). Actitudes culturales respecto a los perros. Ponencia. XII Congreso Nacional Centroamericano de Medicina Veterinaria AnimaNaturalis Internacional, 2014.

Cruz, B. (2015). Psicología canina: la relación de los perros con los humanos. Recuperado de http://www.escuela-canina-rangel.com/uncategorized/ psicologia-canina-la-relacion-de-los-perros-con-los-humanos/

Ministerio de Salud Pública y Bienestar Social. Vice ministerio de Salud Pública. Dirección de Vigilancia de la Salud. Servicio Nacional de Erradicación del Paludismo. Programa Nacional de Control de las Leishmaniosis. (2011). Manual de diagnóstico y tratamiento de las leishmaniosis. Asunción: MSPyBS.

Miret, J., Medina, M., Velázquez, A. L., Sosa, L. \& Castagnino, M. (2011). Leishmaniasis visceral en caninos errantes en la ciudad de Asunción: Rev. Parag. Epidemiol., 2(2), 13-22. 